\section{Evaluación del perfil de eficacia y seguridad de vildagliptina en vida real de pacientes chilenos con diabetes mellitus tipo 2}

\author{
GONZALO GODOY J. ${ }^{1}$, VICENTE GUTIÉRREZ ${ }^{2}$, \\ MARIO MONTECINOS ${ }^{3}$, ALEJANDRO YENES ${ }^{4}$
}

\section{Safety and efficacy of Vildagliptin in real life Chilean diabetic patients}

Background: Vildagliptin is a dipeptidyl peptidase IV inhibitor (DPP4i). Its efficacy and safety of DPP4i in Chilean real life type 2 diabetic (T2D) patients is not well known. Aim: To assess the safety profile and effectiveness of 12 weeks of treatment with Vildagliptin for glycemic control in T2D Chilean patients with a poor glycemic control. Patients and Methods: Retrospective assessment of the effects of Vildagliptin treatment during 12 weeks in 103 T2D patients aged 29 to 92 years ( $47 \%$ males). The main outcomes were changes in glycosylated hemoglobin and the occurrence of adverse effects. Results: After 12 weeks of Vildagliptin use, glycosylated hemoglobin decreased from $8.3 \pm 1.4$ to $7.2 \pm 1.1 \%(p<0.01)$. Fasting plasma glucose and the number of hypoglycemic events also decreased significantly. No significant weight change was observed. The treatment had good compliance, tolerance and patient satisfaction. Conclusions: Vildagliptin treatment reduced glycosylated hemoglobin by $1.1 \%$ and was well tolerated in this group of diabetic patients.

(Rev Med Chile 2015; 143: 63-68)

Key words: Adamantane; Hypoglycemic agents; Pragmatic clinical trials as topics.

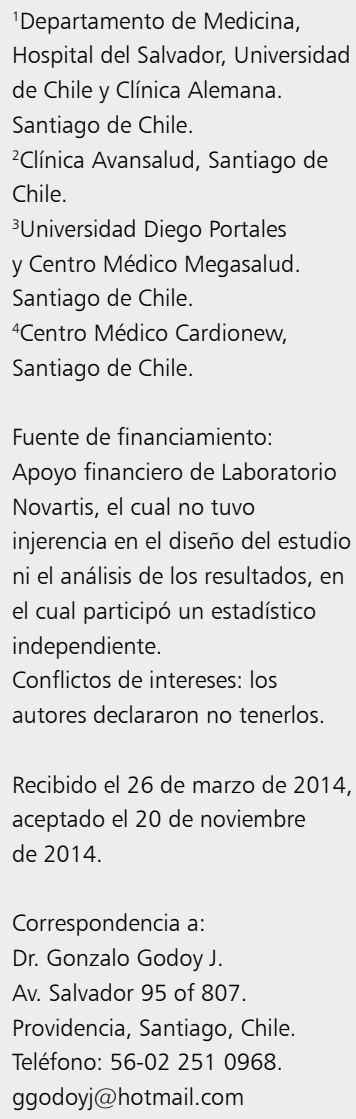

E n la era de la medicina basada en la evidencia ${ }^{1}$, los médicos requieren evaluar los resultados de estudios clínicos controlados usando estudios en condiciones de vida real, que permitan tomar decisiones de prescripción eficaces y con un perfil de seguridad adecuado. Los estudios clínicos controlados miden la eficacia de una intervención, eliminando la mayor cantidad de variables que puedan interferir con un resultado. Por ello, seleccionan bajo estrictos criterios de inclusión y exclusión, poblaciones específicas con el propósito de objetivar lo más preciso posible, la eficacia de la intervención. Sin embargo, la práctica clínica habitual difiere de estas condiciones, requiriéndose conocer en la población enferma habitual con comorbilidades, medicamentos concomitantes, e inserta en un ambiente determinado, la efectividad de las intervenciones previamente consideradas como eficaces ${ }^{2}$. Debido a ello, los estudios observacionales han ido tomando importancia progresiva como fuente de evidencia y validación de resultados clínicos, demostrando que los resultados terapéuticos pueden variar al ser aplicados en vida real y a poblaciones distintas de las ya estudiadas.

Vildagliptina es un hipoglicemiante oral de la clase de los inhibidores de la dipeptidil peptidasa 4 (iDPP4) que ha demostrado su eficacia y seguridad en estudios clínicos controlados internacionales ${ }^{3,4}$. Sin embargo, hasta la fecha desconocíamos los 
efectos en vida real de los iDPP4 en población chilena. Este estudio retrospectivo, evalúa en vida real el control glicémico, seguridad y tolerancia de vildagliptina, en pacientes con diabetes mellitus tipo 2 (DMT2) chilenos, que no habían logrado buen control glicémico con dieta y/o ejercicio y/o uno o dos hipoglicemiantes orales de otra clase.

\section{Pacientes y Método}

Estudio observacional, retrospectivo, en pacientes DMT2 chilenos, con mal control glicémico a pesar de dieta y/o ejercicio y/o uno o dos hipoglicemiantes orales, en quienes su médico tratante había indicado vildagliptina de acuerdo a su juicio clínico, un mínimo de 6 meses previo al inicio del estudio. Se evaluó el control glicémico con el uso de vildagliptina $50 \mathrm{mg}$ dos veces al día, después de al menos 12 semanas de tratamiento.

El objetivo primario fue evaluar el control glicémico a través de hemoglobina glicosilada A1c (HbAlc), previo y posterior al uso de vildagliptina en pacientes DMT2, considerando significativos cambios de $-0,5 \% \mathrm{HbA} 1 \mathrm{c}$ desde el control basal a 12 semanas con vildagliptina.

Los objetivos secundarios fueron: a) Comparar los eventos hipoglicémicos y glicemia de ayuno con vildagliptina versus los tratamientos hipoglicemiantes previos; b) cambio de peso con vildagliptina; c) análisis de subpoblaciones por edad, nivel de Hb1Ac basal e índice de masa corporal (IMC) y d) tolerancia y satisfacción del paciente.

La seguridad se evaluó en términos de eventos adversos registrados durante el período que recibieron vildagliptina.

Se seleccionaron pacientes con DMT2 mayores de 18 años con datos confiables de al menos 6 meses previos y 3 meses posteriores al inicio de vildagliptina. Se exigió un mínimo de 2 evaluaciones médicas para cada paciente (pre y post cambio de terapia) de acuerdo a la práctica clínica habitual, con una HbAlc basal y a 3 meses ( \pm 2 semanas) del inicio de vildagliptina. Se excluyeron pacientes en tratamiento con insulina, uso de iDPP4 previo al uso de vildagliptina, pacientes con insuficiencia cardíaca, respiratoria, hepática y renal severas, historia de cetoacidosis diabética o estado hiperglicémico hiperosmolar no cetósico, diabetes mellitus tipo 1 , embarazo y lactancia. Se obtuvieron de la ficha clínica los datos demográficos basales y terapéuticos: edad, género, peso, estatura, IMC, duración de diabetes, presión arterial (PA), tratamientos previos, exámenes de laboratorio, complicaciones diabéticas, comorbilidades y tratamientos, eventos adversos a medicamentos.

El protocolo fue revisado y autorizado por los Comités de Ética Científicos del Servicio de Salud Metropolitano Oriente y el Servicio de Salud Metropolitano Sur, previo a la revisión de fichas médicas. El investigador firmó acuerdo para conducir el estudio según protocolo y normas de calidad presentadas a los Comités de Ética y autoridades regulatorias.

El tamaño de la muestra se calculó para el objetivo primario del estudio con un poder de $90 \%$, desviación estándar de $\sigma=1,5$ usando un test $t$ de doble cola $\alpha=0,05$. Las diferencias en hipoglicemia y peso desde la basal, se realizaron usando test t de doble cola. Se calculó el intervalo de confianza de $95 \%$ doble cola para la diferencia estimada.

Los eventos adversos se compararon con sus controles históricos.

Los datos de laboratorio se resumieron usando rangos normales y cambios desde las basales (promedios, medianas, desviaciones estándar).

\section{Resultados}

\section{Datos demográficos}

Se revisaron las fichas clínicas de 115 pacientes, tratados en 4 centros ambulatorios de Santiago; 105 con datos completos antes del inicio de vildagliptina. Se retiraron 2 pacientes que usaron insulina (desviación de protocolo).

La edad promedio de los pacientes fue 62,8 años (rango: 29-92 años), 66\% era mayor de 60 años y $47 \%$ de género masculino. El tiempo promedio de diagnóstico de DMT2 fue de 6 años. Sesenta y seis por ciento de los pacientes tenía antecedentes familiares de DMT2. El IMC promedio, previo al uso de la droga del estudio, fue de $30 \mathrm{Kg} / \mathrm{m}^{2}$.

\section{Tratamiento previo de la DMT2}

El 80\% de los pacientes declaró seguir algún tipo de dieta en forma regular, y $38 \%$ ejercicio (no definidos previamente).

Once por ciento de los pacientes estaba sin hipoglicemiantes orales, $40 \%$ con monoterapia, 
correspondiendo en $89 \%$ a metformina. Cuarenta y siete por ciento de los pacientes estaba con biterapia, 95\% de ella correspondió a metformina con sulfonilurea. Siete por ciento de los pacientes recibía otros medicamentos hipoglicemiantes (pioglitazona, glinida). Todos los pacientes, salvo uno, recibieron vildagliptina asociada a metformina y $29 \%$ tuvo una sulfonilurea asociada a la droga de estudio.

\section{Comorbilidades}

El $71 \%$ de los pacientes tenía hipertensión arterial, definida por una PA $>130 / 80,72 \%$ dislipidemia, $43 \%$ tenía complicaciones diabéticas previas; $37 \%$ microvasculares (nefrológicas $23 \%$, neurológicas $11 \%$, de retina $16 \%$ ) y $20 \%$ macrovasculares ( $8 \%$ cardiovascular, $3 \%$ cerebrovascular, $6 \%$ enfermedad arterial periférica), $20 \%$ tenía microalbuminuria y $64 \%$ otras comorbilidades.

\section{Objetivo primario}

La HbAlc promedio previa al inicio del tratamiento con vildagliptina fue $8,3 \pm 1,4 \%$. Después de 12 semanas de tratamiento, la HbAlc fue 7,2 $\pm 1,1 \%$, diferencia estadísticamente significativa $(\mathrm{p}<0,00001)$ (Figura 1).

Con el iDPP4, 25\% de los pacientes alcanzó $\mathrm{HbAlc}<6,5 \%$ y $52 \%$ HbAlc $<7,0 \%$, las que previo al uso de vildagliptina eran 2 y $15 \%$ respectivamente.

\section{Objetivos secundarios:}

La glicemia en ayunas y los eventos de hipoglicemia disminuyeron significativamente con el uso de vildagliptina (Tabla 1).

El peso promedio previo y posterior al tratamiento no tuvo diferencias significativas con el uso de vildagliptina ( 80 y $79 \mathrm{~kg}$ respectivamente).

\section{Análisis de subpoblaciones:}

Se comparó población mayor y menor de 60 años (definidas como adulta y adulta mayor) para permitir el análisis estadístico.

Destaca la importante caída de HbAlc en menores de 60 años ( $-1,6 \%$, versus- $0,9 \%$ en mayores de 60 años). Los menores de 60 años, previo a vildagliptina tenían $\mathrm{HbAlc}$, glicemia de ayuno y peso de mayor magnitud que en adultos mayores (Tabla 2). La disminución de los eventos hipoglicémicos posterior al uso de vildagliptina en DMT2 mayores de 60 años fue de 9,3 veces. En menores de 60 años, la disminución de hipoglicemias fue de 3,6 veces.

\section{Perfil de seguridad}

Los eventos adversos reportados fueron 4 con el uso de vildagliptina ( 3 hipoglicemias y 1 caso de meteorismo). No se reportaron eventos adversos graves.

La tolerancia a vildagliptina, evaluada por los pacientes, fue buena (99\%), al igual que la adherencia $(88,5 \%)$ y satisfacción del paciente $(94,2 \%)$.

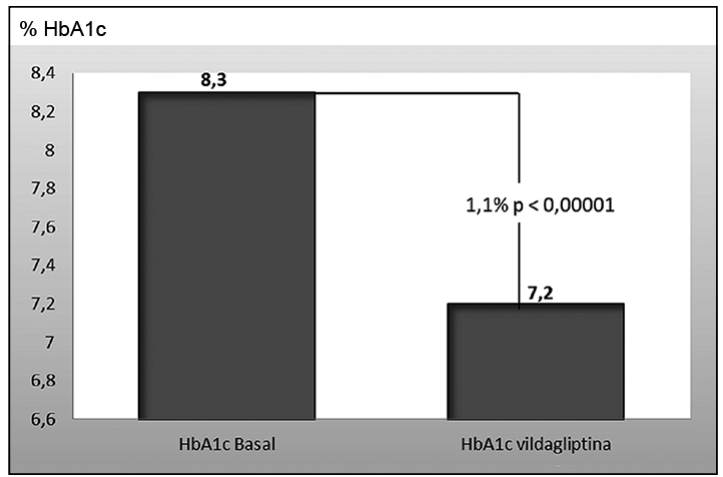

Figura 1. Hemoglobina glicosilada basal y con uso de vildagliptina al menos 12 semanas en DMT2 chilenos.

Tabla 1. Variaciones de hemoglobina glicosilada, eventos hipoglicémicos, glicemia de ayuno y peso en 103 pacientes DMT2 sometidos al uso de vildagliptina por al menos 12 semanas

\begin{tabular}{|lccc|}
\hline Medición & Previo a vildagliptina & Con vildagliptina & p \\
HbA1c (\%) promedio & 8,3 & 7,2 & $<0,00001$ \\
\% hipoglicemia en DMT2 & 22 & 3 & $<0,00001$ \\
Glicemia de ayuno promedio $(\mathrm{mg} / \mathrm{dl})$ & 178 & 133 & $<0,00001$ \\
Peso promedio $(\mathrm{kg})$ & 80 & 79 & NS \\
\hline
\end{tabular}


Tabla 2. Control glicémico por grupo etario: menores y mayores de 60 años

\begin{tabular}{|c|c|c|c|}
\hline \multirow[b]{2}{*}{ Medición } & \multicolumn{3}{|c|}{ Análisis de subpoblaciones por edad } \\
\hline & Edad (años) & Previa a vildagliptina & Con vildagliptina \\
\hline \multirow[t]{2}{*}{$\mathrm{HbA1c}(\%)$} & Menores de 60 & 8,9 & 7,3 \\
\hline & Mayor o igual a 60 & 8,0 & 7,1 \\
\hline \multirow[t]{2}{*}{ Hipoglicemia (\%) } & Menores de 60 & 11 & 3 \\
\hline & Mayor o igual a 60 & 28 & 3 \\
\hline \multirow[t]{2}{*}{ Glicemia ayuno (mg/dl) } & Menores de 60 & 200 & 132 \\
\hline & Mayor o igual a 60 & 166 & 132 \\
\hline \multirow[t]{2}{*}{ Peso $(\mathrm{Kg})$} & Menores de 60 & 86,6 & 85,4 \\
\hline & Mayor o igual a 60 & 77,1 & 75,4 \\
\hline
\end{tabular}

El porcentaje de pacientes que discontinuó tratamiento fue $2 \%$. La discontinuación obedeció a motivos económicos (costo de vildagliptina).

\section{Discusión}

Los pacientes chilenos que recibieron vildagliptina, en $80 \%$ declararon seguir algún tipo de dieta y $38 \%$ ejercicio previo al cambio de terapia, sin embargo, ellos no alcanzan sus objetivos en peso, IMC, ni control glicémico. Esto muestra el alto porcentaje de fracasos en las modificaciones en el estilo de vida como terapia única en el logro de objetivo terapéutico de la DMT2 en vida real, aun cuando los pacientes consideren, en su mayoría, estar siguiendo al menos parcialmente las indicaciones. Además, $88 \%$ de los pacientes estaba recibiendo al menos un hipoglicemiante previo al cambio a vildagliptina, lo que a los 6 años promedio de enfermedad, parece insuficiente para el control glicémico, al menos con las terapias más clásicas de metformina y sulfonilureas que fueron las más frecuentemente utilizadas, y que se agregaban a las indicaciones de cambios en el estilo de vida.

El $66 \%$ de los pacientes tenía antecedentes familiares de diabetes, que pueden corresponder a una carga genética específica, pero las condiciones ambientales también favorecen el desarrollo de la enfermedad (alimentarias, y sedentarismo, que se expresan entre otros, en el sobrepeso y obesidad de esta población, con IMC promedio de 30). Ambas situaciones: la carga genética y el sobrepeso, han sido largamente involucradas en la patogénesis de la enfermedad ${ }^{5}$. De los análisis de subpoblaciones destaca el mayor peso e IMC en los DMT2 más jóvenes, con HbAlc basales más altas respecto de los mayores de 60 años. La población más joven chilena, ha subido de peso antes y en mayor magnitud que la adulta mayor.

Las complicaciones diabéticas, factores de riesgo cardiovasculares y comorbilidades están dentro de lo esperado para la edad y tiempo de evolución de la enfermedad ${ }^{6}$.

El bajo número de eventos adversos y no existencia de eventos adversos serios, debe considerar que estos suelen estar sub-representados en estudios retrospectivos ${ }^{7}$.

La buena tolerancia, adherencia y satisfacción a la terapia con vildagliptina, representa una ventaja terapéutica de esta clase de drogas, y de vildagliptina en particular ${ }^{8}$.

Este estudio en vida real muestra que en pacientes chilenos ambulatorios, vildagliptina mejoró en forma significativa la HbAlc a 12 semanas, con una disminución de HbAlc de -1,1\% ( $p \leq 0,0001)$. Debe destacarse que la HbAlc basal promedio fue $8,3 \%$, lo que motivó el cambio de la terapia hipoglicemiante. Este cambio de $\mathrm{HbAlc}$ coincide con lo encontrado en otros estudios clínicos controlados al agregar vildagliptina ${ }^{3,4,9}$. El estudio en vida real EDGE ${ }^{10}$ con más de 45.000 pacientes DMT2 en terapia dual con vildagliptina y comparadores, muestra una caída de HbAlc de $-1,19 \%$ en la población tratada con vildagliptina, lo que es consistente con lo encontrado en este estudio.

El cambio significativo en hipoglicemias descrito con vildagliptina en la literatura ${ }^{11,12}$ 
también se observa en este estudio, y podría ser consecuencia del alto porcentaje de pacientes que se encontraba con sulfonilurea (SU) asociada a metformina o en monoterapia antes de agregar vildagliptina. De los 23 pacientes con hipoglicemia previo a vildagliptina, 20 estaban con glibenclamida, 1 con repaglinida y 1 estaba sin tratamiento. Actualmente se conoce el alto riesgo de hipoglicemia con sulfonilurea, particularmente con glibenclamida, la SU más usada en Chile ${ }^{13}$. Ello, en contraposición con el bajo número de eventos hipoglicémicos encontrados con iDPP4, en estudios comparativos con $\mathrm{SU}^{11,12}$.

La falta de cambio significativo de peso desde la basal, también confirma lo descrito en la literatura con $\mathrm{iDPP} 4^{14}$, que aparecen como hipoglicemiantes con efecto neutro sobre el peso, a diferencia de lo que ocurre con SU e insulina que producen aumento de peso en el tiempo ${ }^{9-14}$. En una enfermedad de prevalencia creciente como lo es la DMT2 en nuestro país y en el mundo ${ }^{15}$, de carácter progresivo y crónico, se requiere de una terapia efectiva, con la menor cantidad de eventos adversos posibles para lograr mejor adherencia y control a largo plazo, de manera de minimizar las complicaciones micro y macrovasculares ${ }^{16,17}$. Vildagliptina muestra en este estudio de vida real un perfil de eficacia y seguridad adecuado y consistente, probablemente mejorando la funcionalidad de las células pancreáticas ${ }^{18-19} \mathrm{y}$ con cambios en metabolismo glicémico, convirtiéndose así en una alternativa terapéutica oral para los DMT2 chilenos.

\section{Limitaciones del estudio}

Es un estudio en una población y región limitada, que no permite hacer extrapolaciones generales. Sin embargo, su consistencia con los grandes estudios realizados con vildagliptina permiten afirmar la tendencia de los resultados ${ }^{10}$. El análisis de subpoblaciones tiene un carácter más bien descriptivo, debido al bajo número de pacientes en cada subgrupo.

\section{Conclusiones}

Vildagliptina permite un mejor control glicémico, con una disminución de HbA1c de $-1,1 \%$ en los pacientes DMT2 chilenos en vida real estudiados. Ello se asocia a disminución significativa de hipoglicemias, glicemia de ayuno, sin cambios significativos de peso, ni eventos adversos serios. Los pacientes mantienen buena adherencia, reportando buena tolerancia y satisfacción durante el período de uso.

\section{Referencias}

1. Letelier LM, Moore Ph. La medicina basada en evidencia. Visión después de una década. Rev Med Chile 2003; 131: 939-46.

2. Udell JA, Wang TA, Li S, Kohli P, Roe MT, de Lemos JA, et al. Clinical Trial Participation After Myocardial Infarction in a National Cardiovascular Data Registry. JAMA 2014; 312 (8): 841-3.

3. Schweizer A, Couturier A, Foley JE, Dejager S. Comparison between vildagliptin and metformin to sustain reductions in HbAlc over 1 year in drug-naive patients with Type 2 diabetes. Diabet Med 2007; 24: 955-61.

4. Bosi E, Dotta F, Jia Y. Goodman, Vildagliptin plus metformin combination therapy provides superior glycemic control to individual monotherapy in treatment-naive patients with type 2 diabetes mellitus M. Diabetes Obes Metab 2009; 11: 506-15.

5. Meigs JB, Shrader P, Sullivan LM, McAteer JB, Fox CS, Dupuis J, et al. Genotype score in addition to common risk factors for prediction of type 2 diabetes. $\mathrm{N}$ Engl J Med 2008; 359 (21): 2208-19.

6. Liebl A, Mata M, Eschwege E. Evaluation of risk factors for development of complications in Type II diabetes in Europe. Diabetologia 2002; 45: S23-8.

7. Hazell L, Shakir SA. Under-reporting of adverse drug reactions: a systematic review. Drug Saf 2006; 29: $385-$ 96.

8. Schweizer A, Dejager S, Foley JE, Kothny W. Assessing the general safety and tolerability of vildagliptin: value of pooled analyses from a large safety database versus evaluation of individual studies. Vasc Health Risk Manag 2011; 7: 49-57.

9. Stamataros G, Schneider SH. Vildagliptin in the treatment of type 2 diabetes Mellitus. Expert Opin Pharmacother 2011; 12 (12): 1967-73.

10. Mathieu C, Barnett AH, Brath H, Conget I, de Castro JJ, Goke R, et al. Effectiveness and tolerability of secondline therapy withvildagliptin vs other oral agents in type 2 diabetes: Areal-life worldwide observational study (EDGE). Int J Clin Pract doi: 10.1111/ijcp.12252.

11. Ferrannini E, Fonseca V, Zinman B, Matthews D, Ahren B, Byiers C, et al. Fifty-two-week efficacy and safety of vildagliptin vs glimepiride in patients with type 2 diabetes mellitus inadequately controlled on metformin 
monotherapy. Diabetes, Obesity and Metabolism 2009; 11: 157-66.

12. Filozof C, Gautier JF. A comparison of efficacy and safety of vildagliptin and gliclazide in combination with metformin in patient with Type 2 diabetes inadequately controlled with metformin alone: a 52-week, randomized study. Diabet Med 2010; 27: 318-26.

13. Tayek J. SUR receptor activity vs incidence of hypoglycaemia and cardiovascular mortality with sulphonylurea therapy for diabetics. Diabetes Obes Metab 2008; 10: 1128-30.

14. Forst T, Bramlage P. Vildagliptin, a DPP-4 inhibitor for the twice-daily treatment of type 2 diabetes mellitus with or without metformin. Expert Opin Pharmacother 2014; 15 (9): 1299-313.

15. Wild S, Roglic G, Green A, Sicree R, King H. Global prevalence of diabetes: estimates for the year 2000 and projections for 2030. Diabetes Care 2004; 27: 1047-53.

16. Rathmann W, Kostev K, Gruenberger JB, Dworak M,
Bader G, Giani G. Treatment persistence, hypoglycaemia and clinical outcomes in type 2 diabetes patients with dipeptidyl peptidase- 4 inhibitors and sulphonylureas: a primary care database analysis. Diabetes Obes Metab 2013; 15 (1): 55-61.

17. Stratton IM, Adler AI, Neil HA, Matthews DR, Manley SE, Cull CA, et al. On behalf of the UK Prospective Diabetes Study Group Association of glycaemia with macrovascular and microvascular complications of type 2 diabetes (UKPDS 35): prospective observational study. BMJ 2000; 321: 405-12.

18. Ristic S, Bates PC. Vildagliptin: a novel DPP-4 inhibitor with pancreatic islet enhancement activity for treatment of patients with type 2 diabetes. Drugs Today (Barc) 2006; 42: 519-31.

19. Ahren B, Schweizer A, Dejager S, Villhauer EB, Dunning BE, Foley JE. Mechanisms of action of the dipeptidyl peptidase-4 inhibitor vildagliptin in humans. Diabetes Obes Metab 2011; 13: 775-83. 